\title{
EVALUACIÓN DE IMPACTO DE LA EDUCACIÓN SOBRE LA POBREZA EN COSTA RICA (UN ANÁLISIS PARA EDUCACIÓN SECUNDARIA Y POST-SECUNDARIA)
}

\author{
Carmen Sánchez Retana ${ }^{1}$
}

\section{RESUMEN}

En este estudio se evalúa el impacto de la educación sobre la pobreza en Costa Rica. Específicamente, cómo influye la educación secundaria como máximo nivel educativo alcanzado, $y$ la posibilidad de obtener estudios post-secundarios, sobre la probabilidad de sufrir pobreza. Por medio de la metodología Propensity Score Matching y los datos de la Encuesta Nacional de Hogares 2013, se muestra un efecto positivo de la educación en la reducción de la pobreza. Se concluye que, cuando una persona promedio cuenta con educación secundaria completa, reduce la probabilidad de encontrarse en pobreza en 5,7 puntos porcentuales, lo que representa una disminución en las tasas de pobreza de quienes han finalizado secundaria de $21,1 \%$. Por otra parte, la posibilidad de realizar estudios postsecundarios disminuye la probabilidad de pobreza en 8,4 puntos porcentuales, equivalente a una disminución de $36,8 \%$ en las tasas de pobreza de los individuos que al menos han terminado la educación secundaria.

PALABRAS CLAVE: ENSEÑANZA SECUNDARIA, ENSEÑANZA SUPERIOR, MERCADO DE TRABAJO, INGRESOS, PRODUCTIVIDAD, SALARIO, DESEMPLEO, DESIGUALDAD SOCIAL, TASA DE MATRICULACIÓN, ESCOLARIDAD.

\section{ABSTRACT}

This research evaluates the impact of education on poverty in Costa Rica. Specifically, the impact on the probability of being poor of a person that finishes secondary education (highest educational level attained). Furthermore, the impact for a person which has at least this level of education (possibility of post- secondary studies). Using Propensity Score Matching methodology and the survey Encuesta Nacional de Hogares 2013, results show positive impact of education in reducing poverty. When an average person completes the full secondary education reduces the probability of being in poverty by 5.7 percentage points, representing a decrease in poverty rates of those who have completed secondary in $21.1 \%$. Meanwhile, with the possibility of post-secondary studies, the probability is

1 Universidad de Costa Rica. Instituto de Investigaciones en Ciencias Económicas, Código Postal 11501-2060 - Costa Rica; carmen.sanchezretana@ucr.ac.cr 
reduced by 8.4 percentage points. Amount equivalent to a $36.8 \%$ decline in poverty rate of individuals who have at least completed secondary education.

KEYWORDS: SECONDARY EDUCATION, HIGHER EDUCATION, LABOUR MARKET, INCOME, PRODUCTIVITY, WAGES, UNEMPLOYMENT, HUMAN CAPITAL, SOCIAL INEQUALITY, ENROLMENT RATIO.

\section{INTRODUCCIÓN}

La escolaridad de una sociedad es determinante para la erradicación de la pobreza. No existe programa social alguno con mayor impacto a largo plazo en el crecimiento económico y en la reducción de la pobreza como el provocado por las inversiones en educación. Estas constituyen uno de los mejores caminos a tomar bajo el objetivo de ofrecerles niveles decentes de ingreso a las personas pobres y que, de esta manera, puedan ascender en la jerarquía económica (Becker, 1995).

La pobreza se puede entender como una situación de privación, en donde la insuficiencia de ingresos provoca impedimento en alcanzar la satisfacción de necesidades básicas y por ende lograr cierto nivel de bienestar. Una familia se cataloga como pobre cuando sus ingresos son menores que el costo de los bienes y servicios requeridos para mantener un nivel mínimo de subsistencia (INEC, 2013b).

Por su parte, la educación, es vista por Todaro y Smith (2012) como la capacidad de absorber nuevas tecnologías. Es considerada un bien de inversión que aumenta la probabilidad de que un individuo perciba salarios más elevados en el futuro.

El principal vínculo entre educación y superación de la pobreza es el impacto que el nivel y la calidad de la educación tienen sobre la productividad laboral e ingresos futuros de las personas (Larrañaga, 1997). Dicha relación se puede analizar a través de la teoría del capital humano, la cual señala que mayor educación proporciona más capital humano a los agentes, generándoles una mayor productividad que permite competir por los puestos y los ingresos (Bazdresch, 2001).

Diversos estudios ponen en evidencia la significancia de la educación sobre la pobreza. Por ejemplo, Ordaz (2009), al realizar una evaluación de impacto de la educación en la pobreza de México, argumenta que la educación es un mecanismo trascendental para ayudar a los individuos a salir de pobreza, tanto extrema como moderada. Además, indica que el impacto se eleva a mayores niveles educativos. Por su parte, Verner (2004) muestra que en Paraíba (Brasil) la educación es un factor de alta importancia en la reducción de la pobreza; el bajo nivel educacional provoca bajos niveles de productividad $y$, por lo tanto, se generan bajos ingresos. Asimismo, Appleton (2001), al analizar la relación entre educación y pobreza en Uganda encuentra que para el periodo comprendido entre 1992-2000 se dio una mayor disminución en la pobreza en los hogares con jefes de familia con mayor educación.

Por su parte, en Costa Rica Selligson, Martínez y Trejos (1996) demuestran que la existencia de brechas educativas mantiene a aquellos con un bajo nivel educativo en la pobreza. Si se educa a más gente por más largo tiempo, se logra tanto un aumento en la productividad como una distribución más equitativa de los ingresos. Trejos y Montiel (1999) resaltan la existencia de una relación transparente e íntima entre las inversiones en capital humano y el alivio de la pobreza. Sauma y Trejos (2014) señalan la relación inversa existente entre el nivel educativo de los jefes de hogar y la situación de pobreza.

No obstante, si bien existe una estrecha relación entre la educación y la reducción de la pobreza, cabe resaltar que podría no darse la asociación esperada entre el aumento en la escolaridad de la fuerza de trabajo y la tasa de crecimiento del producto por trabajador. Existen tres posibles razones: ineficiencia del ambiente institucional, de manera que la acumulación de capital humano no implique crecimiento económico; exceso de oferta de la mano de obra calificada, lo 
que provocaría un rendimiento marginal descendente de la educación; y calidad educativa baja, no creando capital humano los años de escolaridad (Pritchett, 2001).

En los últimos años, Costa Rica ha presentado niveles de pobreza mayores al 20\%2 (Sauma y Trejos, 2014). Aunado a estos los hogares y la población con insuficiencia de ingresos se ha caracterizado por presentar, en relación a los no pobres, mayor grado de dependencia económica, mayor cantidad de miembros y de niños, menor calificación para el mercado laboral, tasas de desempleo más altas, mayor probabilidad de jefatura femenina, $y$ más alta participación en actividades primarias, secundarias y de cuenta propia. Estas características están relacionadas, en su mayoría, con la situación laboral experimentada por los miembros de los hogares. Reflejando el hecho de que las oportunidades de trabajo no son iguales para todas las personas. Esto como resultado en gran medida de las diferencias de instrucción. Entre mayor sea el nivel de educación alcanzado por una persona, mayor será el ingreso percibido por esta, y por ende menor será su nivel de pobreza.

En cuanto al estado actual de la educación, si bien el país se caracteriza por su inversión en capital humano (lo cual se refleja en aumentos en la escolaridad, aunque en los últimos años dicho crecimiento ha sido discreto), existen retos por superar, en especial en la educación secundaria, la cual está lejos de alcanzar la universalidad y las coberturas esperadas. La mayoría de los jóvenes no concluye la secundaria y el sistema sigue sin atender a la mitad de la población en edad de asistir al ciclo diversificado, aspectos que provocan rezago educativo nacional (Programa Estado de la Nación -PEN-, 2013). Aspecto aún más preocupante si se considera que el peso de su respectiva población se encuentra en crecimiento ante los cambios demográficos (PEN, 2013).

Dado el escenario que enfrenta el país, aunado al deseo de aclarar cuál es el vínculo entre la educación y los niveles de pobreza de las personas, en esta investigación se evalúa el impacto de la educación en la pobreza nacional.

Si bien en Costa Rica existen estudios que muestran la educación como factor incidente en la pobreza (Arguedas, Medaglia y Molina, 2011; Barrios, 2006; Hayes, 2004; Slon y Zúñiga, 2004; entre otros) no se ha realizado una evaluación de impacto como la propuesta en este trabajo. Mientras que las investigaciones relacionadas a la temática se han basado principalmente en la búsqueda y explicación de los determinantes de los niveles de pobreza (donde incluyen la educación dentro de los factores asociados), la presente se enfoca en aislar el efecto que tiene la educación sobre la pobreza, específicamente en estimar el impacto en la probabilidad de sufrir pobreza si se finaliza la educación secundaria y por otro lado, el de concluir al menos dicho nivel educativo (es decir, bajo la posibilidad de que se cuente con estudios post-secundarios).

El utilizar la educación secundaria completa como umbral educativo permite evaluar el impacto en la pobreza de un ciclo académico que se encuentra rezagado, a nivel nacional, respecto a la primaria. Además, se torna más atractivo analizar un nivel educativo caracterizado por un mayor impacto en la pobreza, en relación con los grados de instrucción inferiores a este (Ordaz, 2009, y Verner, 2004).

La determinación del impacto se realiza por medio de la metodología Propensity Score Matching, la cual se enfoca en la estimación de efectos de tratamiento. Esta técnica consiste en encontrar dentro del grupo de no participantes en el tratamiento individuos que sean similares - en términos de características observadas- a los participantes (Khandker, Koolwal y Samad, 2010). En este caso, el tratamiento es el respectivo nivel educativo analizado.

Una vez establecido el emparejamiento se definen algoritmos que permiten realizar la comparación entre los resultados de los tratados con los no tratados, $y$ de esta manera estimar el impacto del tratamiento.

En las estimaciones se utilizan los datos de la Encuesta Nacional de Hogares 2013, los cuales permiten obtener resultados que demuestran la significancia de la educación como mecanismo para

2 Según la metodología Línea de Pobreza aplicada por el INEC. 
reducir la probabilidad de caer en pobreza. Aspecto que, a su vez, se refleja con el hecho de que conforme el nivel de instrucción aumenta, disminuye dicha probabilidad.

Específicamente se estima que, cuando una persona promedio cuenta con educación secundaria completa, reduce su probabilidad de encontrarse en pobreza en 5,7 puntos porcentuales, lo que representa una disminución en las tasas de pobreza de quienes han finalizado secundaria de 21,1\%. Además, ante la posibilidad de obtener estudios post-secundarios, se reduce la probabilidad en 8,4 puntos porcentuales, lo que equivale a una disminución en las tasas de pobreza de 36,8\% para los individuos que al menos han terminado la educación secundaria.

Se evidencia el papel de la educación en la pobreza costarricense, tanto como una herramienta que reduce el riesgo de encontrarse en una situación de ingresos insuficientes, como un camino para salir de ella y experimentar movilidad social.

A continuación se presenta un apartado metodológico que explica la modelación econométrica seguida y los datos utilizados. Posteriormente, en la sección III se muestran los resultados de las diferentes estimaciones correspondientes a la evaluación de impacto. En el apartado IV se mencionan las conclusiones del estudio realizado. Por último, se presentan las referencias utilizadas en la investigación; $y$ en anexos se describen las variables utilizadas en las estimaciones.

\section{METODOLOGÍA}

Se evalúa el impacto que tiene sobre la probabilidad de sufrir pobreza el que una persona:

a. Finalice secundaria.

b. Finalice secundaria o pueda alcanzar un nivel educativo superior a educación secundaria completa.

Los datos utilizados provienen de la Encuesta Nacional de Hogares (ENAHO) realizada por el Instituto Nacional de Estadística y Censos (INEC, 2013a) en julio del año 2013. Esta encuesta contiene información sobre la situación socioeconómica de las personas y sus hogares, a nivel nacional y regional.

Para el análisis, la muestra se restringió a individuos entre 25 y 60 años de edad. Después de los 25 años de edad las personas deberían haber concluido la educación secundaria; y aquellos con deseos de educación post-secundaria ya deberían haberse involucrado en dichos estudios.

Además, se escogió dicho rango etario ante el deseo de analizar un grupo poblacional más económicamente activo- hay muchos inactivos entre los de menor de edad que continúan estudiando y entre los de mayor edad que son dependientes.

Por otro lado, respecto a la metodología utilizada para realizar la evaluación de impacto, se analizó el efecto de tratamiento sobre un grupo de individuos de la muestra, a partir de su comparación con un grupo de control -grupo de individuos similares en ciertas características observables pero que no reciben tratamiento-. En esta evaluación, el tratamiento es en un primer caso contar con la educación secundaria completa, y en un segundo caso, con al menos esta.

Respecto a las características analizadas de los individuos, se dividen en tres categorías: personales, de vivienda y del hogar. Esto con el objetivo de tener una especificación completa de cada sujeto, que permita la obtención de resultados robustos.

Para efectos de la explicación metodológica, el tratamiento es denominado D y el vector de características observables X.

Al comparar la probabilidad de sufrir pobreza en individuos similares, la diferencia entre aquellos que recibieron el tratamiento y aquellos que no, refleja el respectivo impacto de la educación en la imposibilidad de satisfacer las necesidades básicas. Esto permite identificar si, al contar 
con un mayor nivel educativo, un individuo reduce su nivel de pobreza o la probabilidad de caer en dicha situación.

Con el propósito de evitar un posible sesgo de selección durante la escogencia del grupo de control y el de tratamiento, se emplea el método del Propensity Score Matching (PSM), por destacarse como la metodología idónea en este tipo de estudios. Este método, según Ordaz (2009), ha sido diseñado para estimar efectos causales de tratamiento con datos no experimentales o cuando la asignación al grupo de tratamiento está determinada por un número grande de variables.

Además, se debe considerar que la población menos pobre puede decidir acceder a mayor educación. El enfoque semiparamétrico, basado en la metodología del Propensity Score Matching, controla esta situación; pues tiene capacidad de lidiar con el potencial problema de que el resultado de tratamiento esté condicionado por las características no aleatorias de la selección entre los individuos tratados y no tratados. Ante esto, la endogeneidad no debe ser una preocupación en este caso. Al implementar PSM, se generan resultados más precisos en comparación a los que se obtendrían con otras modelaciones.

\section{Metodología Propensity Score Matching}

Consiste en un emparejamiento de las observaciones de control con las observaciones tratadas, basado en la probabilidad del individuo de recibir el tratamiento. Según Khandker, Koolwal y Samad (2010), la idea del PSM es encontrar dentro del grupo de no participantes, individuos que sean similares (en términos de características observadas) a los participantes.

Así mismo, de acuerdo con Rodríguez (2012),"El Propensity Score Matching (PSM), es un algoritmo que empareja participantes y no participantes en un programa en base a la probabilidad condicional de participar (PS), dada una serie de características observables..." (p. 12).

El proceso de encontrar pares asegura que los dos grupos son iguales en los factores observables que se consideran importantes. En este sentido, Rodríguez (2012) indica que:

El PSM es una de las innovaciones más importantes en el desarrollo aplicado de los métodos de emparejamiento, resuelve el problema de la dimensionalidad sintetizando toda la información que proporcionan múltiples variables en una variable única, permitiendo realizar el matching con sola dimensión (p.12).

Una vez establecido el emparejamiento, se define un algoritmo para comparar entre los resultados de los tratados con los no tratados, y de esta manera, estimar el impacto del tratamiento.

Ordaz (2009) acude a esta metodología para evaluar el impacto de la educación primaria y de la secundaria en la pobreza de la zona rural en México. Dicha modelación permitió demostrar que la educación es un mecanismo importante para ayudar a los individuos a salir de pobreza.

A continuación se explican los pasos de la metodología:

\section{a) Estimación del valor o puntaje de probabilidad (Propensity Score)}

Según Rosenbaum y Rubin (1984), el Propensity Score es un valor numérico entre cero y uno, que resume la probabilidad de recibir el tratamiento dado un conjunto de características anteriores a este. La similitud de los individuos se refleja en dicho valor.

Se modela estadísticamente la participación en el programa o tratamiento y luego se calcula la probabilidad de participar dadas sus características de elegibilidad para los individuos de ambas muestras (de control como de tratados). 
Para la obtención del Propensity Score, es necesario unir las muestras de los participantes con los no participantes, con base en el vector de variables $\mathrm{X}$, que se considere determinante para alcanzar el tratamiento (secundaria concluida y posibilidad de estudios post-segundarios). En este estudio, ambas muestras pertenecen a una misma fuente de datos, aspecto que garantiza mejores resultados, ya que todos los datos estarían siendo creados bajo los mismos parámetros ${ }^{3}$.

Unidas las muestras, se procede a estimar la participación en el tratamiento D con base en el vector de variables X. La probabilidad de participación se estima a partir de un modelo Probit 4 .

La forma funcional de probabilidad de recibir tratamiento está dada por:

$$
p(X)=\operatorname{Pr}\{D=1 \mid X\}
$$

donde:

$\mathrm{D}=1$ : recibir el tratamiento

$\mathrm{X}$ : vector de características del agente

Después de la estimación de la ecuación, se obtiene para todos los individuos los Propensity Score (PS) o probabilidad de participar en el tratamiento.

b) Cumplimiento de condiciones: independencia, balanceo, zona de soporte común

Rosenbaum y Rubin (1983) señalan la importancia del cumplimiento de ciertas condiciones para la obtención de resultados consistentes y creíbles en la evaluación de impacto del tratamiento.

Debe haber certeza del cumplimiento de la hipótesis de soporte común. Es decir, debe existir una zona en la que existen suficientes unidades para hacer comparaciones entre el grupo de tratamiento y el grupo de control. Para esto, se requiere un considerable solapamiento o intersección en la distribución de observables entre el grupo de control y el grupo de tratados. Solo en esta área se pueden hacer inferencias sobre la causalidad. Aquellas observaciones que no se encuentren en ésta área son retiradas de la muestra a utilizar ${ }^{5}$.

Además, se debe cumplir con la propiedad de balanceo, la cual se expresa de la siguiente manera:

$$
D \perp X \mid p(X)
$$

Si esta condición se cumple, las observaciones con igual PS deben tener la misma distribución de características, independientemente de la condición de tratamiento. Ante esto, para

3 Según Heckman, Ichimura y Todd (1997), el sesgo en las estimaciones de PSM puede ser bajo si: a) se puede utilizar la misma fuente de datos para tratados como no tratados; b) la muestra es representativa; c) si al utilizar muestras diferentes, estas son muy parecidas.

4 Se podría estimar también con un modelo Logit. Según Gujarati y Porter (2010) los dos modelos son muy semejantes, la principal diferencia es que la distribución logística tiene extremos ligeramente más anchos. Por lo tanto, no existe razón de peso para preferir uno en relación al otro.

5 Cabe resaltar que si, como resultado en la estimación del PS, existen unidades que no reciben nunca el tratamiento o que siempre lo reciben, no existe contrafactual para ellos. No pueden emparejarse con nadie del otro grupo. 
un PS dado, la asignación de tratamiento es aleatoria, cada individuo tiene la misma probabilidad de ser asignado a este (Rosenbaum y Rubin, 1983).

Rosenbaum y Rubin (1983), además, señalan como necesario para identificar el efecto de tratamiento, el supuesto de independencia condicional, también conocido como selección en observables. Es decir, los factores no observables no afectan la participación. Esta condición supone una importante restricción de ortogonalidad entre los posibles resultados y el estado de tratamiento, dadas las variables observadas. De manera formal:

$$
Y_{1} Y_{0} \perp D \mid X
$$

Es decir, la participación, condicionada por las características observables, es independiente de los posibles resultados. En otras palabras, después de controlar por X, las observaciones que se trataron $y$ las que no tienen la misma esperanza del resultado.

c) Estimación efecto promedio de tratamiento en los tratados (ATT). Matching entre participantes y no participantes

Una vez estimado el PS y cumplidas las condiciones necesarias, según Becker e Ichino (2002), se puede estimar el Efecto Promedio de Tratamiento en los Tratados (ATT) de la siguiente forma:

$\mathrm{ATT}=\mathrm{E}\left\{\mathrm{Y}_{1 \mathrm{i}} \mathrm{Y}_{0 \mathrm{i}} \mid \mathrm{D}_{\mathrm{i}}=1\right\}$

$\mathrm{ATT}=\mathrm{E}\left\{\mathrm{Y}_{1 \mathrm{i}}-\mathrm{Y} 0_{\mathrm{i}} \mid \mathrm{D}_{\mathrm{i}}=1, \mathrm{p}(\mathrm{X})\right\}$

$\mathrm{ATT}=\mathrm{E}\left\{\mathrm{E}\left\{\mathrm{Y}_{1 \mathrm{i}} \mid \mathrm{D}_{\mathrm{i}}=1, \mathrm{p}\left(\mathrm{X}_{\mathrm{i}}\right)\right\}-\mathrm{E}\left\{\mathrm{Y}_{0 \mathrm{i}} \mid \mathrm{D}_{\mathrm{i}}=0, \mathrm{p}\left(\mathrm{X}_{\mathrm{i}}\right)\right\} \mathrm{D}_{\mathrm{i}}=1\right\}$

donde:

$\mathrm{Y}_{1 \mathrm{i}}$ : resultado si el individuo es tratado.

$\mathrm{Y}_{0 \mathrm{i}}$ : resultado si el individuo no es tratado.

El valor del ATT indica el impacto de la educación en la pobreza. Para su estimación, se recurre a la metodología de matching. El matching es un método cuasi-experimental de evaluación de impacto, cuyo objetivo principal es establecer un grupo de control para el grupo de tratamiento. Existen diferentes métodos o algoritmos para realizar el emparejamiento de los individuos tratados con los no tratados (según el PS estimado) y de esta manera estimar el ATT. De acuerdo con Becker e Ichino (2002), los más utilizados son Nearest Neighbor Matching, Radius Matching, Kernel Matching, Stratification Matching.

En este trabajo se realiza la evaluación utilizando los algoritmos:

-Matching con el vecino más cercano (Nearest Neighbor Matching): En este se compara el resultado que obtiene cada individuo tratado con el individuo de control cuyo puntaje o valor de probabilidad (Propensity Score) sea el más cercano. Para esto, se establece una cantidad de unidades de control que se emparejan con cada una de las tratadas. Se calcula la diferencia entre cada par de unidades emparejadas en la variable que nos interesa medir $y$, finalmente, se obtiene el impacto sobre la pobreza como el promedio de todas estas diferencias. En este caso, se define si el matching es con reemplazo o sin él; es decir, si cada unidad de control se empareja solo una vez o si puede emparejarse con varias tratadas. 
La fórmula del estimador se define de la siguiente manera:

$$
\begin{gathered}
\text { ATT }=\frac{1}{N^{T}} \sum_{i \in T}\left[Y_{i}^{T}-\sum_{j \in C(i)} w_{i j} Y_{j}^{c}\right] \\
\text { ATT }=\frac{1}{N^{T}} \sum_{i \in T}\left[Y_{i}^{T}-\sum_{i \in T} \sum_{j \in C(i)} w_{i j} Y_{j}^{c}\right]
\end{gathered}
$$

Donde $N^{T}$ es el número de unidades tratadas en la muestra, $C(i)$ corresponde al conjunto de unidades de control emparejadas a la unidad tratada $\mathrm{i}$, con un valor estimado del PS $\mathrm{p}_{\mathrm{i}}$, y las ponderaciones $\mathrm{w}_{\mathrm{ij}}=\frac{1}{N_{i}^{c}}$ si $\mathrm{j} \in \mathrm{C}(\mathrm{i})$ y $\mathrm{w}_{\mathrm{ij}}$ de otra forma. Siendo C (i) $=\min _{j} \| p_{i}-p_{j}$ || (Ordaz, 2009).

-Estratificación (Stratification Matching): Este método consiste en dividir el rango de la variación del puntaje de probabilidad en intervalos, de forma tal que, dentro de cada uno, las unidades de control y tratamiento tengan en promedio el mismo valor o puntaje de probabilidad. El impacto del programa es el promedio ponderado del impacto de todos los intervalos. El ponderador que se utiliza es el porcentaje de tratados dentro de cada intervalo.

Dentro de cada intervalo, la diferencia entre los resultados promedio de los individuos tratados y los de control es estimada de la siguiente manera:

$$
\operatorname{ATT}_{\mathrm{q}} \mathrm{S}=\frac{\sum_{\mathrm{i \in I}(q)} Y_{I}^{T}}{N_{q}^{T}}-\frac{\sum_{\mathrm{j} \in[(q)} Y_{I}^{C}}{N_{q}^{C}}
$$

Siendo I (q) el grupo de unidades en el estrato q.

$N_{q}^{C}$ y $N_{q}^{T}$ son los números de unidades de control y tratadas en el estrato q, respectivamente. Siendo el Q la cantidad total de estratos (Ordaz, 2009).

\section{RESULTADOS}

Como se ha dicho con anterioridad, la evaluación permite identificar el efecto en la probabilidad de caer en situación de pobreza:

a. si se finaliza la educación secundaria (obtención del título de bachillerato, ya sea en secundaria académica o técnica);

b. si se alcanza al menos la finalización de la educación secundaria.

\section{Estimación Propensity Score Matching}

Se realiza una doble especificación, donde por medio de dos sub-muestras, la variable dependiente en el modelo binomial se expresa en cada caso de la siguiente forma:

$\mathrm{SCa}=1$ si concluyó la educación secundaria (máximo nivel educativo alcanzado). $=0$ si no se ha concluido la educación secundaria.

$\mathrm{SCb}=1$ si cuenta con secundaria completa o un nivel educativo mayor. $=0$ si no se ha concluido la educación secundaria. 
En la primera especificación ( $\mathrm{SCa}$ ), se eliminaron de la muestra aquellas personas con niveles educativos superiores a la secundaria con título de bachiller. Es decir, el análisis se limita a comparar personas que finalizaron la educación secundaria (siendo este su máximo escalón educativo) con personas con niveles educativos menores. En este caso la muestra abarca 14.725 personas.

En la segunda muestra, se compararon las personas con educación mayor o igual a bachiller en educación secundaria con las personas con niveles educativos menores a educación secundaria completa. Siendo la muestra de 17.996 personas.

\section{a) Estimación de los Propensity Scores}

Los Propensity Scores indican la probabilidad que tiene cada individuo de recibir el tratamiento. Para la obtención de estos valores se utiliza en cada una de las especificaciones un conjunto de variables independientes que caractericen al individuo y que además puedan estar relacionados con la decisión de realizar estudios secundarios o post-secundarios.

Las variables explicativas utilizadas inicialmente se subdividen en tres categorías, esto con el objetivo de tener una caracterización completa de cada sujeto, que permita alcanzar un mejor emparejamiento y por ende la obtención de resultados robustos.

- Características personales (sexo, zona, estado civil, condición migratoria y edad).

- Características de vivienda (acceso a servicios básicos, tenencia de computadora, la existencia de piso y de cielo raso en la vivienda, el hacinamiento, el estado físico y calidad de la vivienda, tipo de tenencia de la vivienda).

- Características del hogar (total de personas en el hogar (hombres, mujeres y menores de doce años en el hogar), educación y sexo del jefe de familia).

Este conjunto de variables muestra las características de cada uno de los agentes, refleja el ambiente en el que viven estos e inclusive son proxy de la educación del jefe de hogar.

Inicialmente, la regresión en cada una de las especificaciones fue controlada por el conjunto de variables mencionado con anterioridad; sin embargo, ante el deseo de alcanzar un mejor ajuste en la regresión -basado en los criterios de información estadísticos AIC, BIC , R ajustado y VIF-, se dio un descarte de variables que resultaron ser no significativas ${ }^{6}$ en las diferentes especificaciones (tales como piso de tierra, hacinamiento, sexo del jefe de hogar, condición migratoria y no disponibilidad de servicios) o que causaban problemas de multicolinealidad.

Cabe resaltar que el descarte de ciertas variables no significativas permitió alcanzar el cumplimiento de la propiedad de balanceo, la cual es necesaria para la obtención de resultados consistentes.

Ante este escenario, la ecuación Probit que se utiliza en los diferentes casos presenta la siguiente estructura:

Secundaria completa $=\beta_{0}+\beta_{1}$ Zona $+\beta_{2}$ Edad $+\beta_{3}$ Estado civil $+\beta_{4}$ Servicios básicos óptimos $+\beta_{5}$ Estado físico de la vivienda malo $+\beta_{6}$ Estado físico de la vivienda bueno $+\beta_{7}$ Cielo raso $+\beta_{8}$ Computadora $+\beta_{9}$ Total hombres $+\beta_{10}$ Total menores $12+\varepsilon$

Esta regresión se caracteriza por ser la de mejor ajuste dentro del conjunto de expresiones estimadas para cada una de las especificaciones. Por medio de los resultados de los criterios de información estadísticos señalados, existe certeza de la alta predicción de la ecuación para

6 Para determinar si una variable es significativa, se utilizó el valor $p$, el cual indica la probabilidad de equivocarse al rechazar la hipótesis nula. 
determinar la probabilidad de recibir el tratamiento (propensity score). Además, dichas variables son altamente significativas- al 1\%, 5\% y $10 \%$-, existe significancia global y no hay problemas de multicolinealidad.

A continuación, se presentan los resultados de las estimaciones de los modelos Probit para cada una de las especificaciones:

\section{CUADRO 1}

ESTIMACIÓN DEL MODELO PROBIT PARA LA EDUCACIÓN SECUNDARIA

\begin{tabular}{lcccc}
\hline \multirow{2}{*}{ Variable } & \multicolumn{2}{c}{ Primera especificación $(\mathrm{SCa})$} & \multicolumn{2}{c}{ Segunda especificación $(\mathrm{SCb})$} \\
& Coeficiente & $\mathrm{P}>|\mathrm{z}|$ & Coeficiente & $\mathrm{P}>|\mathrm{z}|$ \\
\hline Zona & 0,360 & 0,000 & 0,404 & 0,000 \\
Edad & $-0,013$ & 0,000 & $-0,019$ & 0,000 \\
Estado civil & $-0,085$ & 0,005 & $-0,180$ & 0,000 \\
Servicios básicos óptimos & 0,269 & 0,000 & 0,263 & 0,000 \\
Mal estado físico de la vivienda & $-0,184$ & 0,001 & $-0,219$ & 0,000 \\
Buen estado físico de la vivienda & 0,170 & 0,000 & 0,311 & 0,000 \\
Cielo raso & 0,271 & 0,000 & 0,392 & 0,000 \\
Computadora & 0,567 & 0,000 & 0,912 & 0,000 \\
Total hombres & $-0,066$ & 0,000 & $-0,112$ & 0,000 \\
Total menores 12 & $-0,049$ & 0,003 & $-0,072$ & 0,000 \\
PSEUDO R ${ }^{2}$ & \multicolumn{4}{c}{0,1167} \\
REGIÓN DE SOPORTE COMÚN & {$[0,00757983,0,5289636]$} & 0,2246 & \\
\hline
\end{tabular}

Fuente: Elaboración propia con base en la ENAHO 2013.

Nota1: El mayor valor de log de Verosimilitud se obtuvo después de cuatro iteraciones para cada caso. Número bajo de iteraciones que refleja buena calidad del modelo.

Nota 2: El modelo cuenta con significancia conjunta de las variables al obtener un p valor conjunto 0 .

Nota 3: Ninguna de las especificaciones enfrenta problemas de multicolinealidad.

Existe una relación positiva entre la probabilidad de recibir el tratamiento y pertenecer a la zona urbana. También, con contar con una vivienda en buen estado físico, con servicios básicos óptimos en la misma, $y$ con computadora.

Por otro lado, dicha relación es inversa con la edad, con el hecho de estar casado o en unión libre y con la cantidad de hombres o niños en el hogar.

\section{b) Matching}

Una vez que se tiene la modelación Probit y, con esto, el valor de probabilidad (pscore) para cada una de las observaciones (en las diferentes especificaciones), se realiza el matching, en donde se establecen unidades de control que se comparan con las unidades tratadas; aspecto que permite estimar el ATT.

Cabe señalar el cumplimiento de propiedades para la realización del emparejamiento. En el cuadro anterior se contemplan las regiones de soporte común para cada uno de los casos. En dichas regiones, respectivamente, se comparan los agentes tratados y los no tratados, con el objetivo de evaluar el impacto. 
También, sobresale que, en todos los casos, las especificaciones cumplen con la propiedad de balanceo ${ }^{7}$.

El eliminar de la regresión variables que no son significativas fue de utilidad para el alcance de dicha propiedad.

Para la estimación del matching, en cada caso se acude a la metodología de Nearest Neighbor $y$ la de Estratificación, con las cuales se establece un promedio simple ${ }^{8}$ entre los resultados de ambas para determinar el ATT promedio. Además, se utiliza el soporte común (common suport) en las estimaciones. Este, como se dijo anteriormente, es el establecimiento de la zona en donde se compara el grupo de tratamiento y el grupo de control.

CUADRO 2

ATT IMPACTO DE LA EDUCACIÓN SECUNDARIA EN LA POBREZA

\begin{tabular}{lcccc}
\hline \multirow{2}{*}{ Método } & \multicolumn{2}{c}{ Primera especificación (SCa) } & \multicolumn{2}{c}{ Segunda especificación (SCb) } \\
& ATT & Estadístico & ATT & Estadístico \\
\hline NEAREST NEIGHBOR & $-0,047$ & $-4,129$ & $-0,073$ & $-7,506$ \\
ESTRATIFICACIÓN & $-0,067$ & $-8,190$ & $-0,095$ & $-14,584$ \\
PROMEDIO & $-0,057$ & & $-0,084$ & \\
\hline
\end{tabular}

Fuente: Elaboración propia con base en la ENAHO 2013.

Nota1: Todos los coeficientes ATT son estadísticamente significativos al presentar un T calculado que en su valor absoluto es mayor al T tabular (a valores menores del 5\%).

Los resultados obtenidos muestran la significancia del impacto de la educación en la pobreza. La obtención de secundaria completa disminuye en promedio 5,7 puntos porcentuales la probabilidad de caer en situación de pobreza. Por su parte, el efecto en el caso de contar con la posibilidad de obtener estudios post-secundarios es de una reducción de 8,4 puntos porcentuales, en promedio.

Sin embargo, como la reducción de puntos porcentuales no permite inferir totalmente si el impacto es alto o bajo, dado que depende de la tasa de pobreza existente en las personas, se estima para cada una de las especificaciones la respectiva tasa de pobreza en el área de soporte común.

Para SCa y SCb, la tasa de pobreza de las personas que pertenecen al área de soporte común es de $27,1 \%$ y $22,9 \%$, respectivamente. Ante esto, contar con educación secundaria reduce la probabilidad de sufrir pobreza en $21,1 \%$, en promedio; mientras que finalizar al menos la educación secundaria la disminuye en $36,8 \%$, en promedio.

En la segunda especificación el impacto sobre la pobreza es mayor que el de la primera. El no detener necesariamente los estudios al terminar la educación secundaria provoca una mayor reducción sobre la probabilidad de encontrarse en pobreza. Entre más educación tenga una persona, mayores serán sus ingresos y menor será su nivel de pobreza.

\section{CONCLUSIONES}

Al realizar la evaluación de impacto, se logró apreciar la significancia de la educación como mecanismo para reducir la pobreza en Costa Rica.

Los resultados muestran que los individuos que logran concluir la educación secundaria reducen la probabilidad de encontrarse en pobreza en aproximadamente 5,7 puntos porcentuales (equivalente a una disminución del $21,1 \%$ en su tasa de pobreza). Además, el que una persona no necesariamente

$7 \quad$ Con el cumplimiento de esta propiedad se tiene la certeza de que los dos grupos (tratado y control) son lo suficientemente similares como para asemejarse a grupos que hubiesen sido escogidos de forma aleatoria.

8 Siguiendo a Ordaz (2009), quien realiza un promedio simple entre los métodos utilizados para así obtener el impacto promedio. 
detenga sus estudios en dicho escalón provoca un mayor impacto, disminuyendo en promedio la probabilidad de caer en pobreza en 8,4 puntos porcentuales, lo que corresponde a una reducción del $36,8 \%$ en la tasa de pobreza de aquellos que al menos finalizaron la enseñanza secundaria.

Se evidenció la importancia de la educación como vía para reducir la probabilidad de encontrarse en pobreza o como mecanismo para salir de una situación de ingresos insuficientes. Una escolaridad elevada aumenta las posibilidades de insertarse, bajo buenas condiciones, al mercado laboral; $y$ de esta manera disminuye el riesgo de sufrir privación material.

Dada la coyuntura nacional y los resultados obtenidos -los cuales muestran la importancia de la educación en la reducción de la pobreza-, se percibe la necesidad de alcanzar la universalidad de la educación secundaria y, a la vez, lograr alta cobertura en esta; teniendo presente que, ante el cambio demográfico, se da un aumento relativo del peso poblacional de los adolescentes, aspecto que exigiría incrementar los esfuerzos para lograr las coberturas deseadas (que aumente dicha población no asegura que aumenta la tasa de matrícula).

Además, ante el objetivo de generar un alto impacto de la educación en la probabilidad de sufrir pobreza, es necesario recalcar la importancia de brindar en el país una educación de calidad que se refleje en acumulación de capital humano; existiendo, a la vez, un mercado laboral que demande mano de obra calificada y ofrezca empleos caracterizados por la calidad y por la remuneración adecuada.

\section{AGRADECIMIENTOS}

Se agradecen los valiosos comentarios del señor Juan Diego Trejos y el señor Manfred Esquivel al trabajo previo del que se desprende este artículo.

\section{REFERENCIAS}

Arguedas, I., Medaglia, C., Molina, E. (2011). Movilidad Social en Costa Rica en el periodo 19902008. (Tesis de Licenciatura). Universidad de Costa Rica, San José. Costa Rica.

Bazdresch, M. (2001). Educación y pobreza: una relación conflictiva. Buenos Aires: Consejo Latinoamericano de Ciencias Sociales (CLACSO).

Barrios, Y. (2006). Determinantes de la pobreza en los hogares con adultos mayores. Costa Rica, 2005. (Tesis de Maestría). Universidad de Costa Rica, San José, Costa Rica.

Becker, G. S. (1995). Human capital and poverty alleviation. Human Resources Development and Operations Policy Working Papers (HROWP), 52. Estados Unidos: Banco Mundial. Recuperado de http://www-wds.worldbank.org/external/default/WDSContentServer/WDSP/IB/1995/03/01/ 000009265_3970702134116/Rendered/PDF/multi0page.pdf.

Becker, S. e Ichino, A. (2002). Estimation of average treatment effects bases on propensity scores. The Stata Journal, 2(4), 358-377. Recuperado de http://www.stata-journal.com/sjpdf. html?articlenum=st 0026 .

Gujarati, D. y Porter, D. (2010). Econometría. (5ª . ed.). México: Mc Graw Hill.

Hayes, L. (2004). Factores asociados a las tendencias recientes de la pobreza en Costa Rica, 20012003. (Tesis de Maestría). Universidad de Costa Rica, San José, Costa Rica.

Heckman, J. J., Ichimura, H., y Todd, P. E. (Octubre, 1997). Matching as an econometric evaluation estimator: evidence from evaluation a job training programme. Review of Economic Studies. 64(4), 605-654. doi:10.2307/2971733.

Instituto Nacional de Estadística y Censos (INEC). (2013a). Encuesta Nacional de Hogares 2013. Ingresos de los hogares muestran brechas entre zonas y regiones. (Comunicado de prensa sobre la ENAHO 2013). San José, Costa Rica: INEC.

Instituto Nacional de Estadística y Censos (INEC) (2013b). Encuesta Nacional de Hogares 
(ENAHO)2013. Creación de Variables. San José, Costa Rica: INEC.

Khandker, S., Koolwal, G. y Samad, H. (2010). Handbook on impact evaluation. quantitative methods and practices. Washington D.C.: Banco Mundial. doi:10.1596/978-0-8213-8028-4.

Larrañaga, O. (1997). Educación y superación de la pobreza en América Latina. Quito, Ecuador: Programa de Naciones Unidas para el Desarrollo (PNUD).

Ordaz, J. L. (2009). México: impacto de la educación en la pobreza rural. (Serie Estudios y Perspectivas 105). México, DF: CEPAL.

Programa Estado de la Nación (PEN), (2013). Resumen cuarto Informe Estado de la Educación (4ª ed.). San José: Programa Estado de la Nación.

Pritchett, L. (2001). Where has all the education gone. The World Bank Economic Review, 15(3), 367-391. Recuperado de http://www-wds.worldbank.org/external/default/WDSContentServer/ WDSP/IB/2013/05/21/000356161_20130521150953/Rendered/PDF/773740JRN02001010the0E ducation0Gone.pdf.

Rodríguez, M. (2012). Técnicas de evaluación de impacto: propensity score matching y aplicaciones de prácticas con Stata. (Doc n2/2012). Madrid, España: Instituto de Estudios Fiscales.

Rosenbaum, P. R. y Rubin, D. B. (Septiembre, 1984). Reducing bias in observational studies using sub classification on the propensity score. Journal of the American Statistical Association, 79(387), 516-524. doi:10.2307/2288398.

Rosenbaum, P. R. $y$ Rubin, D. B. (1983). The central role of the propensity score in observational studies for causal effects. Biometrika, 70(1), 41-55. doi:10.1093/biomet/70.1.41.

Sauma, P. y Trejos, J. D. (Junio, 2014). Reducir la pobreza en Costa Rica es posible. Propuestas para la acción. Serie Cuadernos de Desarrollo Humano. San José, Costa Rica: PNUD. Recuperado de http://www.undp.org/content/dam/costa_rica/docs/undp_cr_reducirpobreza_2014.pdf.

Selligson, M., Martínez, J. y Trejos, J. D. (1996). Reducción de la Pobreza en Costa Rica: el impacto de las políticas públicas. San José, Costa Rica: Instituto de Investigaciones en Ciencias Económicas -Programa de Naciones Unidas para el Desarrollo.

Slon, P. y Zuñiga, E. (2004). Aspectos dinámicos de la pobreza en Costa Rica. (Tesis de Licenciatura). Universidad de Costa Rica. San José, Costa Rica.

Todaro, M. y Smith, S. (2012). Human capital: Education and health in economic development. (11 a . ed.). Estados Unidos: Pearson.

Trejos, J. D. y Montiel, N. (1999). El capital de los pobres en Costa Rica. Acceso, Utilización y Rendimiento. Documento de trabajo R-360. Red de Centros de Investigación de la Oficina del Economista Jefe, Banco Interamericano de Desarrollo.

Verner, D. (2004). Education and its poverty-reducing effects: The case of Paraíba, Brazil. (Research Working Paper 3321). Banco Mundial. doi:10.1596/1813-9450-3321.

ANEXO

DESCRIPCIÓN DE VARIABLES UTILIZADAS

\begin{tabular}{ll}
\hline Nombre de variable & Valores que asume \\
\hline \multirow{2}{*}{ Calidad de vivienda aceptable } & 1 : calidad de vivienda aceptable \\
& 0 : otro \\
Calidad de vivienda mala & $1:$ calidad de vivienda mala \\
& $0:$ otro \\
Calidad de vivienda óptima & $1:$ calidad de vivienda óptima \\
& 0 : otro \\
\hline
\end{tabular}

Continúa... 
Continuación....

\begin{tabular}{|c|c|}
\hline Nombre de variable & Valores que asume \\
\hline \multirow{2}{*}{ Cielo raso } & 1: cielo raso en la vivienda \\
\hline & 0:no hay cielo raso en la vivienda \\
\hline \multirow{3}{*}{ Condición migratoria } & 0 : no migrante \\
\hline & 1: migrante interno \\
\hline & 2: migrante externo \\
\hline \multirow[t]{2}{*}{ Edad } & Variable continua \\
\hline & Variable continua \\
\hline Educación jefe de hogar & $\begin{array}{l}\text { Se refiere a los años de escolaridad del jefe de hogar al que pertenece } \\
\text { cada una de las observaciones. }\end{array}$ \\
\hline \multirow{2}{*}{ Estado civil } & 1: unión libre o casado \\
\hline & 0:otro \\
\hline \multirow{2}{*}{ Buen estado físico de la vivienda } & 1:bueno \\
\hline & 0 : otro \\
\hline \multirow{2}{*}{ Mal estado físico de la vivienda } & 1: malo \\
\hline & 0: otro \\
\hline \multirow{2}{*}{ Regular estado físico de la vivienda } & 1: regular \\
\hline & 0 : otro \\
\hline \multirow{2}{*}{ Hacinamiento de vivienda } & 1: vivienda hacinada \\
\hline & 0 : vivienda no hacinada \\
\hline \multirow{2}{*}{ Migrante externo } & 1: es migrante externo \\
\hline & 0 : otro \\
\hline \multirow{2}{*}{ Migrante interno } & 1: es migrante interno \\
\hline & 0 : otro \\
\hline \multirow{2}{*}{ No migrante } & 1: no es migrante interno ni externo \\
\hline & 0:otro \\
\hline \multirow{2}{*}{ Piso de tierra } & 1:tiene piso de tierra (no tiene piso) \\
\hline & 0 : otro \\
\hline \multirow{3}{*}{ Pobre } & 1:pobre \\
\hline & 0:no pobre \\
\hline & $\begin{array}{l}\text { Se acude a la estimación de la pobreza por medio de la metodología } \\
\text { "línea de pobreza" la cual compara el ingreso per cápita de los hogares } \\
\text { con el costo también per cápita de satisfacción de un grupo de } \\
\text { necesidades básicas, tales como cantidades de alimentos requeridos } \\
\text { por un individuo para satisfacer ciertas necesidades calóricas, vivienda, } \\
\text { vestido, educación, salud, cuidados personales, entro otros. } \\
\text { Para aplicar esta metodología se requiere disponer del cálculo de la } \\
\text { línea de pobreza extrema (que se estima con el valor de la canasta } \\
\text { básica alimentaria), la línea de pobreza total (se estima con la canasta } \\
\text { básica total, la cual además incluye el costo de necesidades básicas no } \\
\text { alimentarias) y el ingreso per cápita de los hogares. La línea de pobreza } \\
\text { se compara con el ingreso per cápita del hogar, lo que permite clasificar } \\
\text { a las personas y a los hogares según su condición: en pobreza extrema, } \\
\text { en pobreza no extrema y no pobres (INEC, 2013b). } \\
\text { En este caso tomará valor } 1 \text { si se encuentra en pobreza no extrema o } \\
\text { extrema; } y \text { valor } 0 \text { si no se encuentra en pobreza. }\end{array}$ \\
\hline
\end{tabular}


Continuación....

\begin{tabular}{|c|c|}
\hline Nombre de variable & Valores que asume \\
\hline \multirow[t]{7}{*}{ Secundaria completa } & SCa: \\
\hline & $\begin{array}{l}\text { 1: si concluyó la educación secundaria (máximo nivel educativo } \\
\text { alcanzado) }\end{array}$ \\
\hline & 0: si no se ha concluido la educación secundaria \\
\hline & SCb: \\
\hline & 1: si cuenta con secundaria finalizada o un nivel educativo mayor \\
\hline & 0: si no se ha concluido la educación secundaria. \\
\hline & 1: no dispone de servicios básicos \\
\hline \multirow[t]{2}{*}{ Servicios básicos } & 2: dispone de servicios básicos deficientes \\
\hline & 3: dispone de servicios básicos óptimos \\
\hline \multirow{2}{*}{ Servicios básicos deficientes } & 1: dispone de servicios básicos deficientes \\
\hline & 0: otro \\
\hline \multirow{2}{*}{ Servicios básicos no disponibles } & 1: no dispones de servicios básicos \\
\hline & 0: otro \\
\hline \multirow{2}{*}{ Servicios básicos óptimos } & 1: dispone de servicios básicos óptimos \\
\hline & 0: otro \\
\hline \multirow{2}{*}{ Sexo } & 1: hombre \\
\hline & 0:mujer \\
\hline \multirow{2}{*}{ Sexo jefe de hogar } & 1: hombre \\
\hline & 0:mujer \\
\hline Total hogar & Variable continua \\
\hline Total hombres & Variable continua \\
\hline Total menores 12 & Variable continua \\
\hline Total mujeres & Variable continua \\
\hline \multirow{2}{*}{ Vivienda propia } & 1: vivienda propia (no endeudada) \\
\hline & 0:otro \\
\hline \multirow{2}{*}{ Zona } & 1: urbano \\
\hline & 0:rural \\
\hline
\end{tabular}

Fuente: Elaboración propia con base a la ENAHO 2013. 
